\title{
A phase II randomized trial of sodium oligomannate in Alzheimer's dementia
}

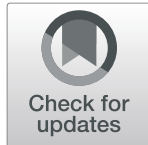

Tao Wang ${ }^{1,2^{*}}$, Weihong Kuang ${ }^{3}$, Wei Chen ${ }^{4}$, Wenwei X ${ }^{5}$, Liming Zhang ${ }^{6}$, Yingjie Li ${ }^{7}$, Hailin Li ${ }^{8}$, Ying Peng ${ }^{9}$, Yangmei Chen ${ }^{10}$, Baojun Wang ${ }^{11}$, Jinsong Xiao ${ }^{12}$, Honghua $\mathrm{Li}^{13}$, Chuanzhu Yan ${ }^{14}$, Yifeng Du ${ }^{15}$, Mouni Tang ${ }^{16}$, Zhiyi He ${ }^{17}$, Haibo Chen ${ }^{18}$, Wei Li ${ }^{19}$, Hong Lin ${ }^{20}$, Shugui Shi ${ }^{21}$, Jianzhong Bi ${ }^{22}$, Huadong Zhou ${ }^{23}$, Yan Cheng ${ }^{24}$, Xiaoping Gao ${ }^{25}$, Yihui Guan ${ }^{26}$, Qiu Huang ${ }^{27}$, Kewei Chen ${ }^{28}$, Xianliang Xin ${ }^{29}$, Jian Ding ${ }^{30}$, Meiyu Geng ${ }^{30^{*}}$ and Shifu Xiao ${ }^{1,2^{*}}$ (i)

\begin{abstract}
Background: Sodium oligomannate (GV-971), a marine-derived oligosaccharide, is a novel agent that may improve cognition in AD patients.

Methods: The 24-week multicenter, randomized, double-blind, placebo parallel controlled clinical trial was conducted in AD in China between 24 October 2011 and 10 July 2013. The study included a 4-week screening/ washout period, followed by a 24-week treatment period. Patients were randomized in a 1:1:1 ratio to receive GV$971900 \mathrm{mg}, 600 \mathrm{mg}$, or placebo capsule in treatment period, respectively. The primary outcome was cognitive improvement as assessed by changes in Alzheimer's Disease Assessment Scale-cognitive subscale 12-item (ADAScog12) scores from baseline to week 24. The secondary efficacy outcomes included CIBIC-Plus, ADCS-ADL, and NPI at 24 weeks after treatment compared with baseline. A subgroup study was assessment of the change in cerebral glucose metabolism by fluorodeoxyglucose positron emission tomography measurements.
\end{abstract}

Results: Comparing with the placebo group ( $n=83$, change -1.45 ), the ADAS-cog 12 score change in the GV-971 600-mg group ( $n=76$ ) was -1.39 ( $p=0.89)$ and the GV-971 900-mg group $(n=83)$ was $-2.58(p=0.30)$. The treatment responders according to CIBIC-Plus assessment were significantly higher in the GV-971 900-mg group than the placebo group $(92.77 \%$ vs. $79.52 \%, p<0.05)$. The GV-971 900-mg subgroup showed a lower decline of cerebral metabolic rate for glucose than the placebo subgroup at the left precuneus, right posterior cingulate, bilateral hippocampus, and bilateral inferior orbital frontal at uncorrected $p=0.05$. The respective rates of treatmentrelated AEs were $5.9 \%, 14.3 \%$, and $3.5 \%$.

Conclusions: GV-971 was safe and well tolerated. GV-971 900 mg was chosen for phase III clinical study.

Trial registration: ClinicalTrials.gov, NCT01453569. Registered on October 18, 2011.

Keywords: Sodium oligomannate, Efficacy, Safety, Alzheimer's disease, Clinical trial

\footnotetext{
*Correspondence: wtshhwy@163.com; mygeng@simm.ac.cn;

xiaoshifu@msn.com

'Department of Geriatric Psychiatry, Shanghai Mental Health Center,

Shanghai Jiaotong University School of Medicine, Shanghai, China

${ }^{30}$ State Key Laboratory of Drug Research, Shanghai Institute of Materia

Medica, Chinese Academy of Sciences, 555 Zu Chong Zhi Road, Shanghai

201203, China

Full list of author information is available at the end of the article
}

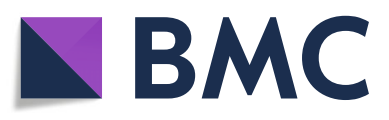

(c) The Author(s). 2020 Open Access This article is licensed under a Creative Commons Attribution 4.0 International License, which permits use, sharing, adaptation, distribution and reproduction in any medium or format, as long as you give appropriate credit to the original author(s) and the source, provide a link to the Creative Commons licence, and indicate if changes were made. The images or other third party material in this article are included in the article's Creative Commons licence, unless indicated otherwise in a credit line to the material. If material is not included in the article's Creative Commons licence and your intended use is not permitted by statutory regulation or exceeds the permitted use, you will need to obtain permission directly from the copyright holder. To view a copy of this licence, visit http://creativecommons.org/licenses/by/4.0/. The Creative Commons Public Domain Dedication waiver (http://creativecommons.org/publicdomain/zero/1.0/) applies to the data made available in this article, unless otherwise stated in a credit line to the data. 


\section{Background}

Globally, approximately 35.6 million individuals live with dementia, and this number is predicted to double by 2030 and more than triple by 2050 [1]. The number of people with Alzheimer's disease (AD) was estimated to be 5.69 million in 2010, with the incidence being 6.25 cases/1000 person-years [2]. The currently available drug treatments for AD target neurotransmitter pathways implicated in disease pathophysiology [3]. While agents such as acetylcholinesterase inhibitors and N-methyl-Daspartic acid receptor antagonists, including memantine, may stabilize or slow the decline of cognition, function, and behavior in patients with $\mathrm{AD}$, they do not slow down the pathological progression [4]. AD is pathologically characterized by senile plaques, neurofibrillary tangles, reactive astrocytosis, and neuronal cell loss. A major component of senile plaques, implicated in the pathophysiology of $A D$, is the aggregated $\beta$-amyloid $(A \beta)$ peptide $[5,6]$. Despite the urgent clinical need, in the past decade, disease-modifying therapies such as those targeting amyloid deposition and tau protein have failed to demonstrate clinically relevant efficacy [7-10].

Certain key amino acid residues and specific domains of the $A \beta$ peptide reportedly play an important and unique role in $A \beta$ aggregation $[11,12]$. Sodium oligomannate (GV-971) is a marine-derived oligosaccharide [13]; by multitargeting various $A \beta$ subregions, it inhibits $A \beta$ aggregation and destabilizes $A \beta$ aggregates into nontoxic conformers. Moreover, GV-971 can evidently reconstitute the dysbiosis of gut microbiota, reduce metabolite-driven peripheral infiltration of immune cells into the brain, and inhibit neuroinflammation [14]. These effects have been reported to protect synapse integrity and improve cognition in vitro and in a transgenic mouse model of AD [15-18].

Herein, we report the results of a phase II trial designed to investigate the optimal dose, efficacy, and safety of GV-971 capsules in patients with mild-tomoderate AD.

\section{Methods}

\section{Study design}

This 24-week, multicenter, randomized, double-blind, placebo-controlled, phase II trial was conducted in patients with mild-to-moderate $\mathrm{AD}$ in geriatric psychiatry, neurology, or geriatrics departments at 24 hospitals (i.e., centers) in China between 24 October 2011 and 10 July 2013. The study included a 4-week screening/washout period, followed by a 24-week treatment period. During the 4-week screening/washout period, all patients received placebo capsules, and during the 24-week treatment period, they underwent the following treatment: three 150-mg GV-971 capsules b.i.d. (900-mg group), two 150-mg GV-971 capsules plus one placebo capsule b.i.d. (600-mg group), or three placebo capsules b.i.d. The trial protocol had two versions: 1.1 and 2.1. The main amendment was the additional clarifications of hypothesis testing in version 2.1 than version 1.1 .

The protocol was registered on https://clinicaltrials. gov/ct2/show/NCT01453569 and approved by the China Food and Drug Administration (approval nos. 2006L02492, 2011L00942). The trial was also approved by the Institutional Review Boards of all participating centers from which the approval of the protocol and all related documents was obtained. The trial protocol can be found in Additional file 1.

\section{Participants}

\section{Inclusion criteria}

Patients aged between 50 and 85 years (inclusive) were eligible to participate in this study, regardless of their gender. They should have been educated to primary school level and above, with the ability to complete pertinent cognitive tests and other rating scales. To qualify for the trial, a subject was expected to fulfill the diagnostic criteria of probable AD according to the National Institute of Neurological and Communicative Disorders and Stroke and the Alzheimer's Disease and Related Disorders Association (1984), with mild-to-moderate stages $(10 \leq$ total Mini-Mental State Examination score $\leq 24)$ $[19,20]$. In addition, the total Hachinski Ischemic Scale [21] score had to be $\leq 4$ and the total 17-item Hamilton Depression Scale [22] score had to be $\leq 10$. Furthermore, it was mandatory for each participant to have stable, reliable caregivers who were expected to be with them for at least 4 days/week for at least $2 \mathrm{~h}$ each time while the patient was awake. The caregivers were required to provide valuable information on the Clinician Interview Based Impression of Change-Plus (CIBIC-Plus) [23], Alzheimer's Disease Cooperative Study-Activities of Daily Living (ADCS-ADL) [24], and Neuropsychiatric Inventory (NPI) $[25,26]$ assessments. Prior to implementation of any protocol-related procedure or examination, the subjects were required to sign a written informed consent form. If they were unable to sign the form due to impaired cognition, their legal guardians were asked to sign on their behalf.

\section{Exclusion criteria}

Subjects were excluded if they had participated in any another clinical trial within 30 days prior to the initiation of this study, if they were pregnant or nursing, or if they had dementia due to non-AD causes. Furthermore, they were excluded if they had abnormal laboratory values, including glutamic pyruvic transaminase or glutamic oxaloacetic transaminase $>1.2$ times the upper limit of normal; creatinine $>1.2$ times the upper limit of normal; white blood cell count, platelet count, or hemoglobin 
level below the lower limit of normal; and blood glucose level $>1.5$ times the upper limit of normal. Patients with systolic blood pressure $\geq 160 \mathrm{mmHg}$ or diastolic blood pressure $\geq 100 \mathrm{mmHg}$ during screening were also not enrolled. In addition, patients were excluded if they had unstable or severe cardiac, pulmonary, hepatic, renal, or hematopoietic disease; a visual or hearing disorder that prevented completion of the neuropsychological test and scale evaluation; and history of alcohol or drug abuse, psychosis (including severe depression), or use of drugs for AD that could not be stopped. Further details pertaining to the inclusion and exclusion criteria have been provided in Additional file 1.

\section{Randomization and masking}

Patients were randomized in a 1:1:1 ratio to receive 900 mg GV-971, $600 \mathrm{mg}$ GV-971 plus placebo, or placebo during the treatment period via an interactive web response system (IWRS) managed by GCP ClinPlus Co., Ltd. (China). All distributed trial drugs had corresponding numbers. Investigators logged on to IWRS for identifying a participant with the help of a center-specific subject ID and visit ID. The system then distributed the drugs to the designated subject using a system-generated drug number.

\section{Study assessments}

Herein, the primary endpoint was cognitive improvement as assessed by changes in Alzheimer's Disease Assessment Scale-cognitive subscale 12-item (ADAS$\operatorname{cog} 12)$ [27] scores from baseline to week 24. The decline of ADAS-cog12 score means the cognitive improvement of patient. The secondary efficacy outcomes included a global assessment based on CIBIC-Plus, along with improvements in ADCS-ADL and NPI scales at 24 weeks post-treatment as compared with baseline data. An assessment of changes in cerebral glucose metabolism, as evaluated by fluorodeoxyglucose positron emission tomography (FDG-PET) measurements of the cerebral metabolic rate for glucose in the brain regions preferentially affected by $\mathrm{AD}$, was also performed in a subgroup of subjects at two sites in Shanghai and Chengdu; the same inclusion and exclusion criteria were used. Safety assessments included evaluations of adverse events (AEs), serious AEs (SAEs), laboratory test results (routine blood and coagulate function tests), electrocardiography data, and vital signs. The study assessment schedule is provided in Additional file 1.

\section{Statistical analysis}

\section{Sample size determination}

Data collection and analysis were independently managed by GCP ClinPlus Co., Ltd. (China). Power Analysis and Sample Size software (NCSS, LLC, Utah, USA) was used to calculate the sample size. The assumed difference in ADAS-cog mean of -2.0 and common standard deviation of 4 gave rise to the effect size of 0.5 . With $80 \%$ power, type I error of 0.05 , and computed effect size of $0.5(\Delta / \delta)$, we estimated that 70 subjects were needed per group to compare the difference in changes in ADAS- $\operatorname{cog} 12$ total scores from baseline to week 24 between the two treatment groups and the placebo group. Considering a drop-off rate of $20 \%, 84$ subjects were expected to be present in each group (total $=252$ in the three groups).

\section{Efficacy endpoints and analyses}

We finally enrolled 255 cases in this study $(n=85,84$, and 86 in the placebo, 600-mg, and 900-mg groups, respectively). Data were analyzed in the intention-to-treat population and are reported for the full analysis set and safety set after database lock on August 15, 2013. All data were analyzed using SAS version 9.2. The difference in changes in ADAS-cog12 total scores from baseline to week 24 between the two treatment groups was analyzed using the analysis of covariate model, with baseline ADAS- $\operatorname{cog} 12$ total scores serving as the covariate. All results have been expressed as least squares adjusted means and $95 \% \mathrm{CI}$.

\section{FDG-PET}

Subjects in the ${ }^{18} \mathrm{~F}$-FDG-PET subgroup underwent PET scanning at baseline and week 24 . Each participant was instructed to fast for at least $6 \mathrm{~h}$ prior to the PET scan and had serum glucose levels between 63 and $99 \mathrm{mg} /$ $100 \mathrm{~mL}$ prior to radiotracer administration. During the 45-min radiotracer uptake period after the patient received an intravenous bolus dose of $220-370 \mathrm{MBq}(6-$ $10 \mathrm{mCi}) \mathrm{FDG}$, he/she rested quietly in a darkened room with eyes open, in the supine position, and with minimal ambient noise before transferred to the scanning room. FDG-PET scan was then performed using the Siemens Biograph PET/CT (Siemens, Germany), a 30-min emission scan, and a 5-min post-emission CT scan. PET images were reconstructed using filtered back projection, correction for radiation attenuation and scatter. Using SPM8 (https://www.fil.ion.ucl.ac.uk/spm/), the images were spatially normalized to the default MNI (Montreal Neurological Institute) brain template for voxel-wise statistical analysis-based general linear model. The relative cerebral metabolic rate for glucose (CMRgl) was calculated as the standardized uptake value ratio, with the whole brain serving as the reference region. For the brain regions known to be affected by $\mathrm{AD}$, the temporal lobe, hippocampus, posterior cingulate, precuneus, and parietal lobe (all bilateral), we extracted the peak voxel values for each of these regions to examine the baseline-24-week CMRgl change differences between the 
placebo and 900-mg groups under the general linear model.

\section{Results}

Baseline characteristics and overview of study subjects In total, 295 subjects were assessed for eligibility; 255 patients were enrolled and randomly assigned into the placebo $(n=85), 600-\mathrm{mg} \quad(n=84)$, and 900-mg $(n=86)$ groups. Patient disposition is shown in Fig. 1. There were 83 patients in the placebo group, 76 in the 600-mg group, and 83 in the 900-mg group who completed the study and were included in the full analysis set. Overall, $32(12.5 \%)$ patients could not continue their participation as they were lost to follow-up, withdrew consent, showed severe complication/symptom deterioration, experienced AEs or allergic reactions, seriously violated the inclusion/exclusion criteria, or showed noncompliance, among other reasons (Fig. 1). There were 7 , 9 , and 9 patients in the placebo, 600-mg, and 900-mg groups, respectively, who underwent ${ }^{18}$ F-FDG-PET scans. Baseline demographics were not statistically different among the groups $(p>0.05)$ (Table 1), and baseline demographics for the FDG-PET subgroups were also not statistically different $(p>0.05)$.

\section{Primary efficacy outcome}

The effect of treatment on the primary outcomes in the three groups was not significantly different. For the primary efficacy outcome of cognitive improvement at week 24, as measured using ADAS- $\operatorname{cog} 12$ total scores, the mean change from the baseline value was -1.45 in the placebo group $(n=83),-1.39$ in the $600-\mathrm{mg}$ group ( $n=76, p=0.89$ in comparison with the placebo group), and -2.58 in the 900-mg group $(n=83, p=0.30$ in comparison with the placebo group) (Table 2 and Fig. 2). The least squares adjusted mean changes and 95\% CI of ADAS-cog12 total scores from baseline to week 24 were as follows: 900 -mg group $=-2.53,95 \% \mathrm{CI},-3.91$ to 1.15 ; 600 -mg group $=-1.34,95 \% \mathrm{CI},-2.88$ to 0.19 ; and placebo group $=-1.50,95 \% \mathrm{CI},-2.97$ to -0.03 . Though not statistically different, change in ADAS- $\operatorname{cog} 12$ scores from baseline to week 24 in the 900-mg group was numerically greater than that in the placebo group.

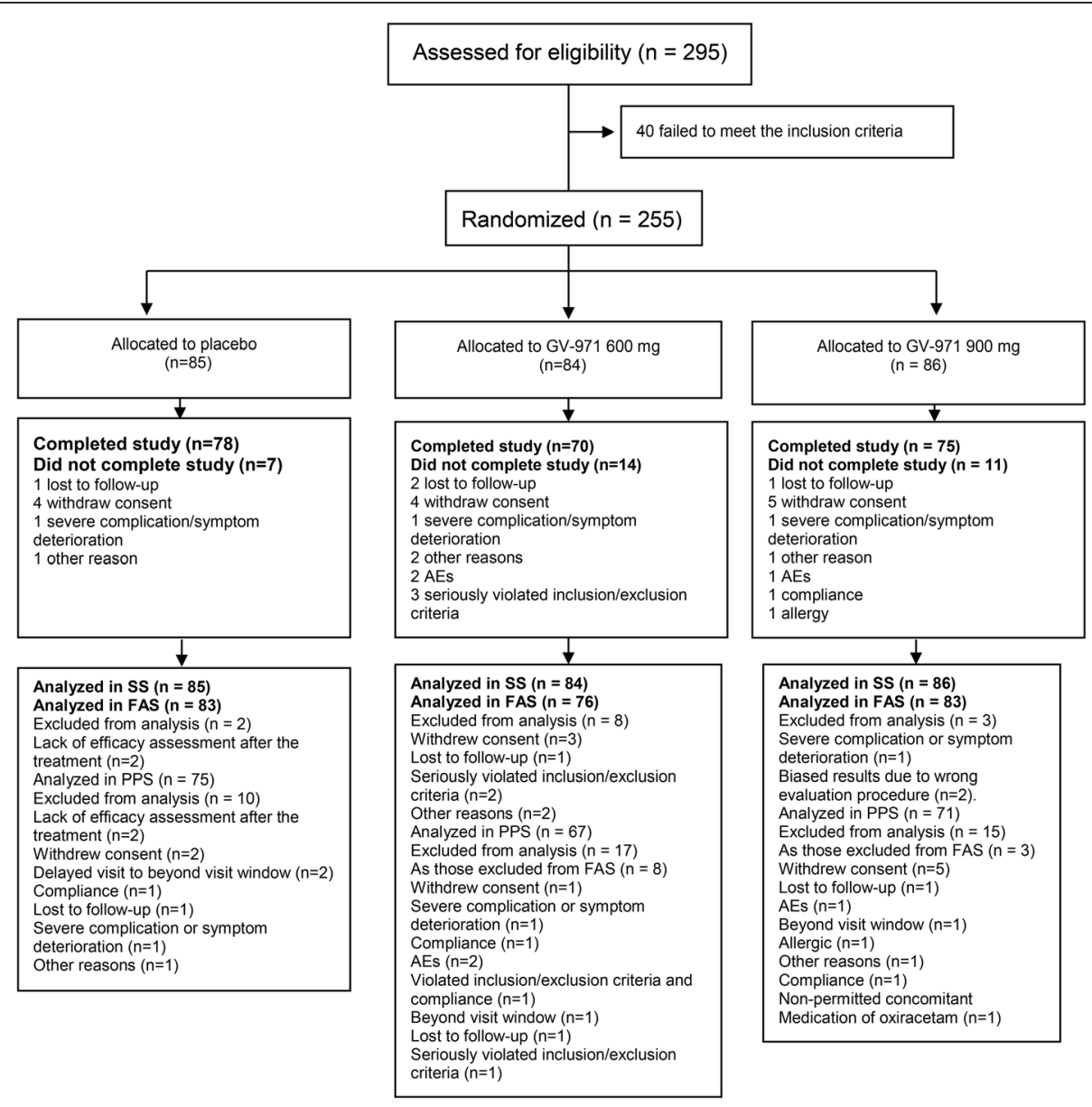

Fig. 1 Patient disposition 
Table 1 Baseline patient characteristics and demographics of full analysis set

\begin{tabular}{|c|c|c|c|c|}
\hline & Placebo $(N=83)$ & $600 \mathrm{mg}(N=76)$ & $900 \mathrm{mg}(N=83)$ & $p$ value \\
\hline Gender & & & & $0.519^{\mathrm{a}}$ \\
\hline Male & $31(37.4 \%)$ & $35(46.1 \%)$ & $33(39.8 \%)$ & \\
\hline Female & $52(62.7 \%)$ & $41(54.0 \%)$ & $50(60.2 \%)$ & \\
\hline Age (years) & $70.3(8.1)$ & $70.3(8.4)$ & $70.4(8.5)$ & $1.00^{\mathrm{b}}$ \\
\hline Ethnicity & & & & $0.76^{\mathrm{a}}$ \\
\hline Han Chinese & $83(100.0 \%)$ & $75(98.7 \%)$ & $82(98.8 \%)$ & \\
\hline Others & $0(0.0 \%)$ & $1(1.3 \%)$ & $1(1.2 \%)$ & \\
\hline Height $(\mathrm{cm})$ & $161.7(8.8)$ & $163.1(7.2)$ & $162.5(7.6)$ & $0.52^{\mathrm{b}}$ \\
\hline Weight (kg) & $58(10.4)$ & $60(9.9)$ & $59(10.0)$ & $0.20^{\mathrm{b}}$ \\
\hline Education & & & & $0.80^{\mathrm{a}}$ \\
\hline 6 years & $31(37.4 \%)$ & $22(29.0 \%)$ & $27(32.5 \%)$ & \\
\hline More than 6 years & $52(62.7 \%)$ & $54(71.1 \%)$ & $56(67.5 \%)$ & \\
\hline Breathing (times/minute) & $18(2.0)$ & $18(2.1)$ & $18(2.1)$ & $0.54^{\mathrm{b}}$ \\
\hline Diastolic blood pressure $(\mathrm{mmHg})$ & $126(12.0)$ & $124(11.8)$ & $126(11.2)$ & $0.65^{\mathrm{b}}$ \\
\hline Systolic blood pressure $(\mathrm{mmHg})$ & $76(7.0)$ & $78(7.4)$ & $76(8.1)$ & $0.24^{b}$ \\
\hline Pulse (times/minute) & $74(10.0)$ & $75(8.9)$ & $74(8.7)$ & $0.85^{\mathrm{b}}$ \\
\hline HIS & $1.3(0.9)$ & $1.5(0.9)$ & $1.3(0.9)$ & $0.88^{\mathrm{b}}$ \\
\hline HAMD & $3.3(2.7)$ & $3.4(2.8)$ & $3.1(2.7)$ & $0.28^{\mathrm{b}}$ \\
\hline MMSE & $17.5(4.2)$ & $18.3(4.8)$ & $18.1(4.4)$ & $0.20^{\mathrm{b}}$ \\
\hline
\end{tabular}

HIS Hachinski Ischemic Scale, HAMD Hamilton Depression Scale, MMSE Mini-Mental State Examination

aExact Pearson's chi-squared test

${ }^{\mathrm{b}} p$ values were determined using two-tailed $t$ tests

\section{Secondary efficacy outcomes}

The assessment of the secondary efficacy outcomes showed that significantly more patients were classified as treatment responders based on CIBIC-Plus at week 24 in the 900-mg group than in the placebo group (Fig. 3). Treatment responders were defined as those with marked improvement, moderate improvement, minimum improvement, or no change on CIBIC-Plus. The difference in the percentage of treatment responders was significant between the 900-mg and placebo groups (92.8\% vs. $79.5 \% ; p=0.01)$, but insignificant between the $600-\mathrm{mg}$ and placebo groups $(68.4 \%$ vs. $79.5 \%$; $p=0.11)$. For other secondary outcome measures, i.e., the effect of the study drug at week 24 on ADCS-ADL and NPI scales, no significant differences were found between the two treatment groups and the placebo group.

\section{${ }^{18}$ F-FDG-PET scan subgroup exploratory analysis}

The effect of GV-971 on cerebral glucose metabolism, as measured using ${ }^{18}$ F-FDG-PET, was evaluated in 7, 9, and 9 patients from the placebo, 600-mg, and 900-mg groups, respectively. CMRgl change differences, not corrected for multiple comparisons, before and after treatment were noted in the brain regions known to be affected by AD between the placebo and the 900-mg groups which were shown in Fig. 4, with the left precuneus $(p=0.003)$, right posterior cingulate cortex $(p=0.005)$, right hippocampus region $(p=0.006)$, left hippocampus $(p=0.003)$, right inferior orbital frontal $(p=0.02)$, and left inferior orbital frontal $(p=0.0008)$ uncorrected for multiple comparisons. However, none of them is significant after multiple comparison corrections. Also, we did not find the group differences in the right precuneus, left posterior cingulate, and bilateral temporal lobe and parietal lobe even with uncorrected $p=0.05$. Finally, there was no baseline CMRgl difference.

\section{Safety analysis}

The AE profile of GV-971 is summarized in Table 3 and Additional file 2. Most AEs were mild to moderate and needed no treatment. The total rate of AEs was $77.6 \%$, $76.2 \%$, and $59.3 \%$ in the placebo, 600-mg, and $900-\mathrm{mg}$ groups, respectively, while the rates of treatment-related AEs were $5.9 \%, 14.3 \%$, and $3.5 \%$ in the aforementioned groups, respectively. The discontinuation rates due to AEs were $1.2 \%$ in the placebo group, $3.6 \%$ in the $600-\mathrm{mg}$ group, and $3.5 \%$ in the $900-\mathrm{mg}$ group. There were 14 reported SAEs (seen as 15 case-events), including six SAEs in the placebo group (six case-events, $7.1 \%$ ), five in the 600-mg group (six case-events, 6.0\%), and three in the 900-mg group (three case-events, 3.5\%). Thirteen SAEs, 
Table 2 Efficacy analyses for the primary and secondary outcomes with the covariate model of full analysis set

\begin{tabular}{|c|c|c|c|}
\hline & Placebo $(N=83)$, mean $(S D)$ & $600 \mathrm{mg}(N=76)$, mean $(S D)$ & $900 \mathrm{mg}(N=83)$, mean $(S D)$ \\
\hline \multicolumn{4}{|l|}{ ADAS- $\operatorname{cog} 12$} \\
\hline Baseline & $28.1(12.0)$ & $26.1(12.4)$ & $26.16(12.00)$ \\
\hline $24 \mathrm{Ws}$ & $26.7(14.5)$ & $24.7(14.4)$ & $23.6(13.7)$ \\
\hline Change & $-1.5(7.0)$ & $-1.4(6.5)$ & $-2.6(5.7)$ \\
\hline$p$ value $^{a}$ & - & $p=0.89$ & $p=0.30$ \\
\hline \multicolumn{4}{|l|}{ ADCS-ADL } \\
\hline Baseline & $50.9(17.4)$ & $50.0(17.4)$ & $53.7(16.4)$ \\
\hline $24 \mathrm{Ws}$ & $49.8(17.5)$ & $49.5(18.7)$ & $53.2(16.7)$ \\
\hline Change & $-1.1(7.8)$ & $-0.5(7.7)$ & $-0.5(8.3)$ \\
\hline$p$ value $^{a}$ & - & 0.66 & 0.48 \\
\hline \multicolumn{4}{|l|}{ NPI } \\
\hline Baseline & $9.9(13.7)$ & $7.3(10.9)$ & $7.4(12.2)$ \\
\hline $24 \mathrm{Ws}$ & $7.8(11.7)$ & $7.5(12.2)$ & $6.2(10.8)$ \\
\hline Change & $-2.1(9.0)$ & $0.2(7.2)$ & $-1.1(11.2)$ \\
\hline$p$ value $^{a}$ & - & 0.17 & 0.94 \\
\hline $\mathrm{CIBIC}+$ & Cases (\%) & Cases (\%) & Cases (\%) \\
\hline Significant improvement & $3(3.6)$ & $2(2.6)$ & $0(0.0)$ \\
\hline Moderate improvement & $6(7.2)$ & $9(11.8)$ & $13(15.7)$ \\
\hline Slight improvement & $32(38.6)$ & $18(23.7)$ & $29(34.9)$ \\
\hline No change & $25(30.1)$ & $23(30.3)$ & $35(42.2)$ \\
\hline Slight deterioration & $17(20.5)$ & $21(27.6)$ & $4(4.8)$ \\
\hline Moderate deterioration & $0(0.0)$ & $3(4.0)$ & $2(2.4)$ \\
\hline Significant deterioration & $0(0.0)$ & $0(0.0)$ & $0(0.0)$ \\
\hline$p$ value $\mathrm{b}_{\text {,* }}$ & - & 0.11 & 0.01 \\
\hline
\end{tabular}

Ws weeks, ADAS-cog Alzheimer's Disease Assessment Scale-cognitive, ADCS-ADL Alzheimer's Disease Collaborative Study-Activity of Daily Living Scale, NPI Neuropsychiatric Inventory, CIBIC Clinician's Interview-Based Impression of Change

aExact Pearson's chi-squared test

${ }^{\mathrm{b}} p$ values were determined using two-tailed $t$ tests

*Comparison of the percentage of effectiveness in the overall efficacy evaluation (significant improvement + moderate improvement + slight improvement + no change) on the CIBIC-Plus scale of sodium oligomannate capsule and placebo at week 24 of treatment

including four in the 600-mg group, three in the 900-mg group, and six in the placebo group, were evaluated by investigators to be definitely unrelated to the study drug. One SAE, behavioral and psychiatric symptoms of dementia, in the 600-mg group was possibly related to the study drug. The assessment of vital signs and physical examination results yielded essentially no abnormalities or intergroup differences. Laboratory test results and ECG findings were similar between the groups at 24 weeks post-treatment as compared with the baseline data.

\section{Discussion}

Oligomannurarate, a marine-derived oligosaccharide, has been identified as a multi-region binder of $A \beta$. Through its multitargeting of various subregions of $A \beta, G V-971$ inhibits $A \beta$ aggregation and destabilizes $A \beta$ aggregates into non-toxic conformers [15-17]. Other important mechanism of GV-971 is that it can reconstitute the dysbiosis of gut microbiota, reduce metabolite-driven peripheral infiltration of immune cells into the brain, and inhibit neuroinflammation [14]. Studies have shown that GV-971 crosses the blood-brain barrier and appears to have a good tolerability profile. In the phase 1 study in healthy volunteers, the compound can be detected in blood. Gender and age have no effects on the pharmacokinetic (PK) profiles of GV-971. GV-971 was shown to have a dose linear PK profiles in the blood, with a halflife of $\sim 11 \mathrm{~h}$ and a good safety profile. In this phase II study, as compared with placebo, the oral administration of GV-971 at $600 \mathrm{mg}$ or $900 \mathrm{mg} /$ day for 24 weeks did not have any significant positive effects on the primary endpoint in patients with mild-to-moderate AD. However, the trial results provided useful information pertaining to the clinical efficacy of GV-971. No significant effect was detected on the primary endpoint of ADAS$\operatorname{cog} 12$ changes at 24 weeks in the $900-\mathrm{mg}$ group as 


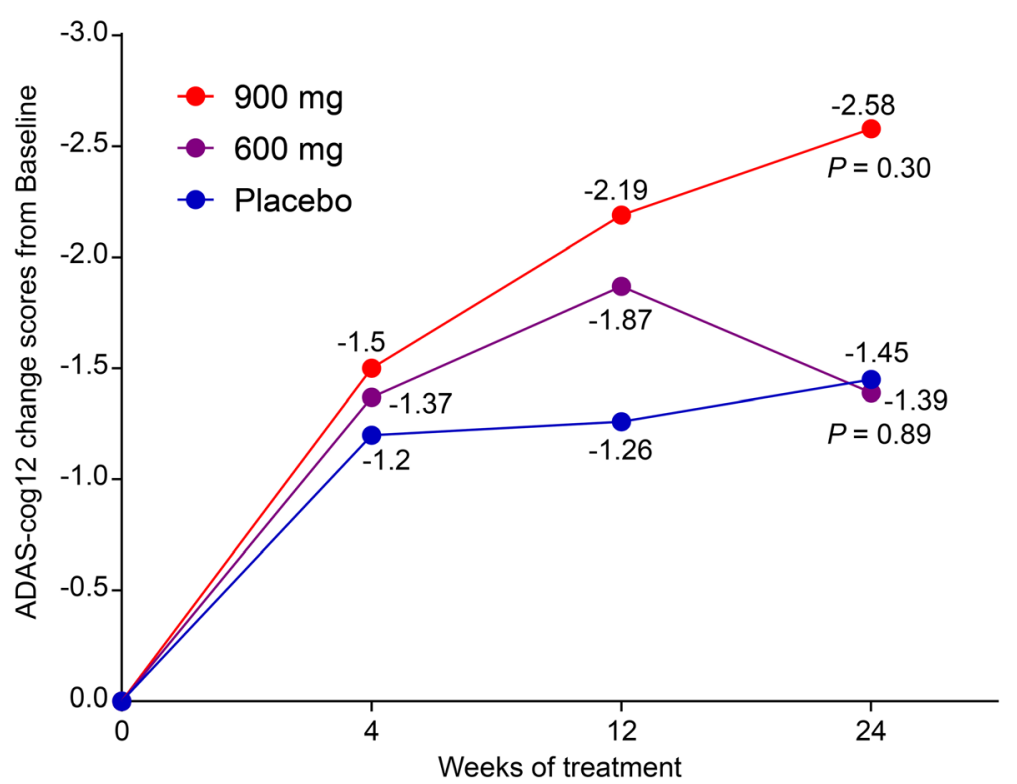

Fig. 2 Full analysis set of the primary efficacy outcome of ADAS-cog12 change scores from baseline to week 24 among the three groups. $p$ values were derived upon comparison of changes from week 24 to baseline with the placebo group

compared to those in the placebo group. The secondary efficacy outcome based on CIBIC-Plus also suggested that administering $900 \mathrm{mg}$ GV-971 improved the global function in patients with mild-to-moderate $\mathrm{AD}$. AD dementia is well known as a progressive disease [1]. We referred to the data processing methods of CIBIC+ in other AD clinical research [28] and considered the no change in the CIBIC+ scale at endpoint as treatment response. The percentage of treatment responders was significantly higher for those in the 900-mg group as compared to those in the placebo group. The placebo effect seemed to appear in nervous system drugs, such as drugs for dementia and depression [29-31]. There have been studies reporting the variation of placebo effects among different racial and ethnic groups in the East Asia [32, 33]. In our case, the unmet cares given by the physicians, in the aspects of the clinical encounter, including the tone and emotional presence of the clinician, and the support and engagement involved in a consultation, have been found to directly shape clinical outcomes. As in the reports, the placebo effect appeared in all of the groups.

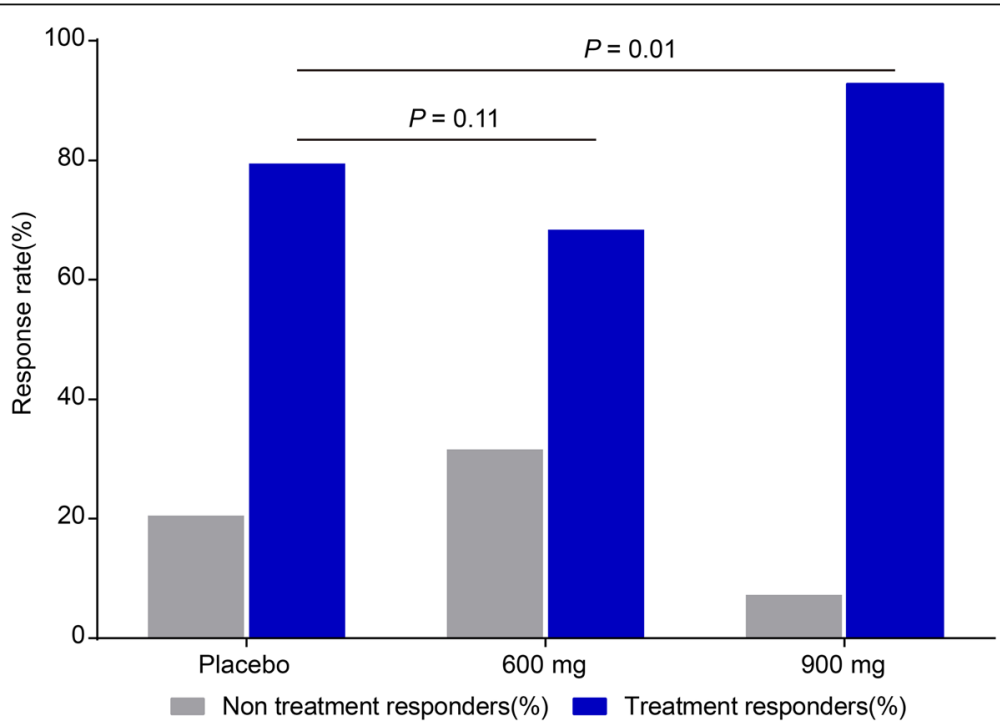

Fig. 3 Full analysis set of the secondary efficacy outcome of CIBIC-Plus change score from baseline to week 24 among groups 


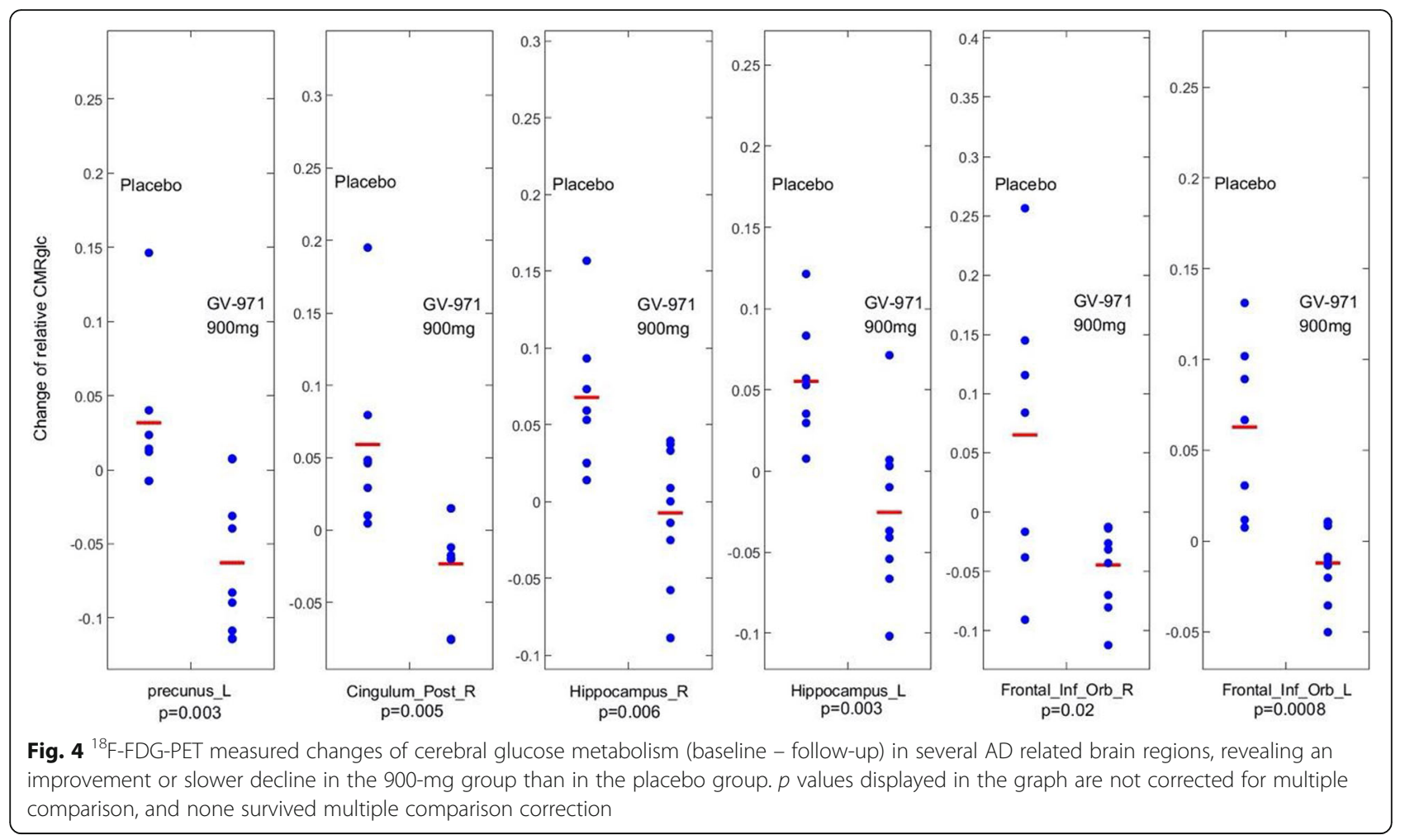

$\mathrm{AD}$ is clinically characterized by progressive cognitive impairment, which is associated with impaired cerebral glucose metabolism [34]. In fact, cerebral glucose hypometabolism occurs during early $\mathrm{AD}$, and ${ }^{18} \mathrm{~F}$-FDG-PET studies have consistently reported progressive reductions in cerebral glucose metabolism, the extent and topography of which correlate with symptom severity [35]. In this 24-week, double-blind, randomized, placebocontrolled trial, we employed FDG-PET to explore the effects of GV-971 on regional neuronal activity in patients with mild-to-moderate AD in a small subcohort of only 7, 9, and 9 subjects from the 3 groups. We acknowledge that the FDG-PET measure was exploratory, so was our analysis which did not survive multiple comparison correction; thus, our findings should be interpreted with caution. ${ }^{18} \mathrm{~F}$-FDG-PET data showed that treatment with GV-971 at $900 \mathrm{mg} /$ day slowed the impairment of cerebral glucose metabolism in several ADassociated brain regions. Given the exploratory nature (including not corrected for multiple comparisons), our results seemed to imply that the effects of GV-971 on neuronal function are not simply compensatory in the brain regions affected by AD.

Treatment with GV-971 was safe and well tolerated. Most AEs were either infections, gastrointestinal disorders, or nervous system disorders. Moreover, as compared with the placebo group, none of the AEs was more prevalent in the active treatment groups. Although

Table 3 Summary of adverse events, serious AEs of all groups

\begin{tabular}{lllll}
\hline Items & $\begin{array}{l}\text { Placebo }(\boldsymbol{N}=\mathbf{8 5}) \\
\text { Cases (\%) }\end{array}$ & $\begin{array}{l}\mathbf{6 0 0} \mathbf{~ m g}(\boldsymbol{N}=\mathbf{8 4}) \\
\text { Cases (\%) }\end{array}$ & $\begin{array}{l}\mathbf{9 0 0} \mathbf{m g}(\boldsymbol{N}=\mathbf{8 6}) \\
\text { Cases (\%) }\end{array}$ \\
\hline AES & $35(41.2)$ & $31(36.9)$ & $24(27.9)$ & $\boldsymbol{p}$ value \\
NR Med & $32(37.7)$ & $26(30.9)$ & $21(24.4)$ & 0.18 \\
RE Med & $3(3.5)$ & $5(6.0)$ & $3(3.5)$ & $3(3.5)$ \\
SAEs & $6(7.1)$ & $5(6.0)$ & $3(3.5)$ & 0.17 \\
NR Med & $6(7.1)$ & $4(4.8)$ & $0(0.0)$ & 0.57 \\
RE Med & $0(0.0)$ & $1(1.2)$ & $3(3.5)$ & 0.56 \\
Drop-off due to AE & $1(1.2)$ & $3(3.6)$ & 0.56 \\
\hline
\end{tabular}

AEs adverse events, SAEs serious AEs, AEs NR Med non-medication-related AEs, AEs RE Med medication-related AEs (definitely, probable, possible, and suspiciously) $p$ values related to $A E$ and $S A E$ were analyzed using the chi-squared test 
the primary outcome measure did not reach statistical significance, findings from this phase II trial are still informative. First, our results suggest that GV-971 dosage should be $900 \mathrm{mg} /$ day whenever a new phase III study is being conducted. Second, no difference of the ADAScog change between the placebo and 900-mg groups suggests that the trial duration should be $>24$ weeks in the future to comprehensively assess the efficacy of GV971.

According to the results of this phase II study, a future phase III trial of GV-971 could be able to reduce the ADAS- $\operatorname{cog} 12$ score from baseline by at least 1.4, which, together with variability information, can be converted to an effect size $(\Delta / \delta)$ of 0.23 . Assuming the two-arm design (placebo vs. $900 \mathrm{mg}$ GV-971), such a study can be conducted with 315 subjects/group with $80 \%$ power to detect differences in the change between the GV-971 and placebo groups for 36-week treatment duration phase III trial, with a two-sided $\alpha$ level of 0.05 .

This phase II study has three important limitations. First, biomarkers associated with AD were not included as a part of the inclusion/exclusion criteria for AD diagnosis at the protocol design and to serve as possible additional output measures. The lack of biomarkers may thus lead to some bias in AD diagnosis. Second, there could have been an evaluation bias at few sites as investigators were not continuously trained to ensure consistency in assessing cognitive test data. Third, there was a potential of continuous improvement in the 900mg group at endpoint, so the 24-week treatment duration may not be long enough to witness the effectiveness of GV-971. Therefore, it is necessary to increase the treatment duration in future phase III trials of GV-971. Last, the cholinesterase inhibitors (ChEIs) have been shown to be effective for treating mild-to-moderate $\mathrm{AD}$. We shall consider the use of ChEIs as a "standard of care" in future GV-971 trial.

\section{Conclusions}

In conclusion, this phase II trial provided the evidence that GV-971 was safe and well tolerated. A decision was made to carry out a phase III clinical trial for GV-971 with the chosen dosage $900 \mathrm{mg}$.

\section{Supplementary information}

Supplementary information accompanies this paper at https://doi.org/10. 1186/s13195-020-00678-3.

Additional file 1.

Additional file 2.

\section{Abbreviations}

GV-971: Sodium oligomannate; AD: Alzheimer's disease; ADAScog12: Alzheimer's Disease Assessment Scale-cognitive subscale 12-item; CIBIC-Plus: Clinician Interview Based Impression of Change-Plus; ADCS-
ADL: Alzheimer's Disease Cooperative Study-Activities of Daily Living; NPI: Neuropsychiatric Inventory; A $\beta$ : $\beta$-Amyloid; b.i.d.: Bis in die; IWRS: Interactive web response system; ID: Identity document; FDGPET: Fluorodeoxyglucose positron emission tomography; AE: Adverse event; SAE: Serious AE; Cl: Confidence interval

\section{Acknowledgements}

The study was sponsored by Shanghai Green Valley Pharmaceutical Co., Ltd. The study was also supported by the National Major Scientific and Technological Special Project (grant 2011ZX09101-003-01) and the National High Technology Research and Development Program of China (grant 2006AA090501). We gratefully acknowledge study investigators and the dedication of study participants.

We thank all the investigators, participants, caregivers, and families who participated in this trial; the site investigators and personnel; the members of the steering committee; and the members of the trial team for their role in the preparation of the manuscript.

\section{Authors' contributions}

SFX was the principal investigator. TW was the subprincipal investigator. SFX, TW, XLX, JD, and MYG were involved in the study design. SFX, TW, WHK, WC, WWX, LMZ, YJL, HLL, YP, YMC, BJW, JSX, HHL, CZY, YFD, MNT, ZYH, HBC, WL, $H L$, SGS, JZB, HDZ, YC, and XPG carried out the study. TW, YHG, XLX, JD, MYG, and SFX were involved in the data analysis and interpretation. $\mathrm{QH}$ and KWC were involved in the FDG-PET data analysis and interpretation. TW drafted the manuscript. All authors critically revised the report, commented on drafts of the manuscript, and approved the final report.

\section{Funding}

The study was sponsored by Shanghai Green Valley Pharmaceutical Co., Ltd. The study was also supported by the National Major Scientific and Technological Special Project (2011ZX09101-003-01) and the National High Technology Research and Development Program of China (2006AA090501) We gratefully acknowledge study investigators and the dedication of study participants.

\section{Availability of data and materials}

The datasets used and/or analyzed during the present study are available from the corresponding author on reasonable request.

\section{Ethics approval and consent to participate}

The trial protocol was approved by the Ethics Review Board of Shanghai Mental Health Center (Shanghai, China). The trial protocol was approved by the Institutional Review Boards of all participating sites. All participants or their representatives provided written informed consent before participation in the trial.

\section{Consent for publication}

All authors approved the final version of the manuscript for submission.

\section{Competing interests}

Shifu Xiao has been a provisional consultant of Pfizer, Lilly, Novartis, GSK, Johnson \& Johnson, Lundbeck, and Green Valley and received honorariums. Kewei Chen was a paid consultant and a full-time employee of Shanghai Green Valley Pharmaceutical Co., Ltd. at the time the FDG-PET data was analyzed, and the time the manuscript was prepared respectively. Currently, he is a full-time employee of Banner Health to which Banner Alzheimer's Institute belongs, and serves as a paid consultant to Shanghai Green Valley Pharmaceutical Co., Ltd. Xianliang Xin is a full-time employee of the Shanghai Green Valley Pharmaceutical Co Ltd. Part of the results of this paper was presented at the 7th Clinical Trials Conference on Alzheimer's Disease (CTAD) November 20-22, 2014, Philadelphia, PA, USA.

\section{Author details}

${ }^{1}$ Department of Geriatric Psychiatry, Shanghai Mental Health Center, Shanghai Jiaotong University School of Medicine, Shanghai, China. ${ }^{2}$ Alzheimer's Disease and Related Disorders Center of Shanghai Jiaotong University, 600 South Wan Ping Road, Shanghai 200030, China. ${ }^{3}$ Department of Psychiatry, West China Hospital of Sichuan University, Chengdu, Sichuan, China. ${ }^{4}$ Department of Neurology, Sir Run Run Shaw Hospital, Affiliated with the Zhejiang University School of Medicine, Hangzhou, Zhejiang, China. 
${ }^{5}$ Department of Geriatric Psychiatry, Wuxi Mental Health Center, Wuxi, Jiangsu, China. ${ }^{6}$ Department of Neurology, First Affiliated Hospital of Harbin Medical University, Harbin, Heilongjiang, China. 'Department of Neurology, The Hospital of 81st Group Army PLA, Zhangjiakou, Hebei, China. ${ }^{8}$ Department of Geriatric Psychiatry, Nanjing Brain Hospital Affiliated to Nanjing Medical University, Nanjing, Jiangsu, China. ${ }^{9}$ Department of Neurology, Sun Yat-Sen Memorial Hospital, Sun Yat-Sen University, Guangzhou, Guangdong, China. ${ }^{10}$ Department of Neurology, The Second Affiliated Hospital of Chongqing Medical University, Chongqing, China.

${ }^{11}$ Department of Neurology, Baotou Central Hospital, Baotou, Inner Mongolia Autonomous Region, China. ${ }^{12}$ Department of Neurology, Zhongnan Hospital of Wuhan University, Wuhan, Hubei, China. ${ }^{13}$ Department of Neurology, Central War Zone General Hospital of the Chinese People's Liberation Army, Wuhan, Hubei, China. ${ }^{14}$ Department of Neurology, Qilu Hospital of Shandong University, Jinan, Shandong, China. ${ }^{15}$ Department of Neurology, Shandong Provincial Hospital, Jinan, Shandong, China. ${ }^{16}$ Department of Geriatric Psychiatry, Guangzhou Brian Hospital, Guangzhou, Guangdong, China. ${ }^{17}$ Department of Neurology, The First Hospital of China Medical University, Shenyang, Liaoning, China. ${ }^{18}$ Department of Neurology, Beijing Hospital, Beijing, China. ${ }^{19}$ Department of Neurology, Shanghai Ninth People's Hospital, Shanghai Jiao Tong University School of Medicine, Shanghai, China. ${ }^{20}$ Department of Neurology, Tangdu Hospital, Air Force Military Medical University, Xi'an, Shanxi, China. ${ }^{21}$ Department of Neurology, The First Hospital Affiliated to AMU (Southwest Hospital), Chongqing, China. ${ }^{22}$ Department of Neurology, The Second Hospital of Shandong University, Jinan, Shandong, China. ${ }^{23}$ Department of Neurology, Daping Hospital, Chongqing, China. ${ }^{24}$ Department of Neurology, Tianjin Medical University general hospital, Tianjin, China. ${ }^{25}$ Department of Neurology, Hunan Provincial People's Hospital, Changsha, Hunan, China. ${ }^{26}$ PET Center Huashan Hospital Fudan University, Shanghai, China. ${ }^{27}$ Med-X Research Institution, Shanghai Jiao Tong University, Shanghai, China. ${ }^{28}$ Banner Alzheimer's Institute, Phoenix, AZ, USA. ${ }^{29}$ Shanghai Green Valley Pharmaceutical Co Ltd, Shanghai, China. ${ }^{30}$ State Key Laboratory of Drug Research, Shanghai Institute of Materia Medica, Chinese Academy of Sciences, 555 Zu Chong Zhi Road, Shanghai 201203, China.

Received: 2 July 2020 Accepted: 2 September 2020

Published online: 14 September 2020

\section{References}

1. World Health Organization. Dementia: a public health priority. Available from: URL: https://www.who.int/mental_health/publications/dementia_ report 2012/en/

2. Chan KY, Wang W, Wu JJ, et al. Epidemiology of Alzheimer's disease and other forms of dementia in China, 1990-2010: a systematic review and analysis. Lancet. 2013;381(9882):2016-23.

3. Tan CC, Yu JT, Wang HF, et al. Efficacy and safety of donepezil, galantamine, rivastigmine, and memantine for the treatment of Alzheimer's disease: a systematic review and meta-analysis. J Alzheimers Dis. 2014:41(2):615-31.

4. NICE National Institute for Health and Care Excellence. Donepezil, galantamine, rivastigmine and memantine for the treatment of Alzheimer's disease. Available from: URL: http://www.nice.org.uk/guidance/TA217/chapter/1-guidance.

5. Gong Y, Chang L, Viola KL, et al. Alzheimer's disease-affected brain: presence of oligomeric A beta ligands (ADDLs) suggests a molecular basis for reversible memory loss. Proc Natl Acad Sci U S A. 2003;100(18):10417-22.

6. Masters $\mathrm{CL}$, Cappai R, Barnham KJ, Villemagne VL. Molecular mechanisms for Alzheimer's disease: implications for neuroimaging and therapeutics. J Neurochem. 2006;97(6):1700-25.

7. Panza F, Solfrizzi V, Imbimbo BP, Logroscino G. Amyloid-directed monoclonal antibodies for the treatment of Alzheimer's disease: the point of no return? Expert Opin Biol Ther. 2014;14(10):1465-76.

8. Castello MA, Jeppson JD, Soriano S. Moving beyond anti-amyloid therapy for the prevention and treatment of Alzheimer's disease. BMC Neurol. 2014;14:169.

9. Doody RS, Thomas RG, Farlow M, et al. Phase 3 trials of solanezumab for mild-to-moderate Alzheimer's disease. N Engl J Med. 2014;370(4):311-21.

10. Salloway $S$, Sperling R, Fox NC, et al. Two phase 3 trials of bapineuzumab in mild-to-moderate Alzheimer's disease. N Engl J Med. 2014;370(4):322-33.

11. Liao MQ, Tzeng YJ, Chang LY, et al. The correlation between neurotoxicity, aggregative ability and secondary structure studied by sequence truncated Abeta peptides. FEBS Lett. 2007;581(6):1161-5.

12. Maji SK, Ogorzalek Loo RR, et al. Amino acid position-specific contributions to amyloid beta-protein oligomerization 2009, 284(35):23580-23591.
13. Wang T. New drug research and development for Alzheimer's pathology: present and prospect. Shanghai Arch Psychiatry. 2017;29(4):171-3.

14. Wang $X$, Sun $G$, Feng $T$, et al. Sodium oligomannate therapeutically remodels gut microbiota and suppresses gut bacterial amino acids-shaped neuroinflammation to inhibit Alzheimer's disease progression. Cell Res. 2019; 29(10):787-803.

15. Kong LN, Geng MY, Mu L, Xin XL, Yang N, Zuo PP. Effects of acidic oligose on differentially expressed genes in the mice model of Alzheimer's disease by microarray. Acta Pharm Sin. 2005;40(12):1105-9.

16. Fan Y, Hu J, Li J, et al. Effect of acidic oligosaccharide sugar chain on scopolamine-induced memory impairment in rats and its related mechanisms. Neurosci Lett. 2005;374(3):222-6.

17. Hu J, Geng M, Li J, et al. Acidic oligosaccharide sugar chain, a marinederived acidic oligosaccharide, inhibits the cytotoxicity and aggregation of amyloid beta protein. J Pharmacol Sci. 2004:95(2):248-55.

18. Jiang RW, Du XG, Zhang $X$, et al. Synthesis and bioassay of beta-(1,4)-Dmannans as potential agents against Alzheimer's disease. Acta Pharmacol Sin. 2013;34(12):1585-91.

19. Folstein MF, Folstein SE, McHugh PR. "Mini-mental state". A practical method for grading the cognitive state of patients for the clinician. J Psychiatr Res. 1975;12:189-98.

20. Katzman R, Zhang MY, Ouang-Ya-Qu, et al. A Chinese version of the MiniMental State Examination; impact of illiteracy in a Shanghai dementia survey. J Clin Epidemiol 1988, 41:971-978.

21. Hachinski VC, lliff LD, Zilhka E, et al. Cerebral blood flow in dementia. Arch Neurol. 1975:32:632-7.

22. Hamilton M. A rating scale for depression. J Neurol Neurosurg Psychiatry. 1960;23:56-62.

23. Boothby $\mathrm{H}$, Mann $\mathrm{AH}$, Barker A. Factors determining inter rater agreement with rating global change in dementia: the cibic-plus. Int J Geriat Psychiatry. 1995:10:1037-45.

24. Galasko D, Bennett D, Sano M, et al. An inventory to assess activities of daily living for clinical trials in Alzheimer's disease. The Alzheimer's Disease Cooperative Study. Alzheimer Dis Assoc Disord. 1997;11(Suppl 2):S33-9.

25. Cumming JL, Mega M, Gray K, Rosenberg-Thompson S, Carusi DA, Gornbein J. The Neuropsychiatric Inventory: comprehensive assessment of psychopathology in dementia. Neurology. 1994:44:2308-14.

26. Wang T, Xiao S, Li X, et al. Reliability and validity of the Chinese version of the neuropsychiatric inventory in mainland China. Int J Geriat Psychiatry. 2012;27:539-44

27. Rosen WG, Mohs RC, Davis KL. A new rating scale for Alzheimer's disease. Am J Psychiatry. 1984;141:1356-64

28. Wilcock GK, Lilienfeld S, Gaens E. Efficacy and safety of galantamine in patients with mild to moderate Alzheimer's disease: multicentre randomised controlled trial. Galantamine International-1 Study Group. BMJ. 2000; 321(7274):1445-9.

29. Rogers SL, Friedhoff LT. The efficacy and safety of donepezil in patients with Alzheimer's disease: results of a US multicentre, randomized, double-blind, placebo-controlled trial. The Donepezil Study Group. Dementia. 1996;7(6):293-303.

30. Salloway S, Sperling R, Fox NC, et al. Two phase 3 trials of bapineuzumab in mild-to-moderate Alzheimer's disease. N Engl J Med. 2014;370(4):322-33.

31. Khan A, Fahl Mar K, Faucett J, Khan Schilling S, Brown WA. Has the rising placebo response impacted antidepressant clinical trial outcome? Data from the US Food and Drug Administration 1987-2013 [published correction appears in World Psychiatry. 2017:16(3):328]. World Psychiatry. 2017:16(2):181-92.

32. Geuter S, Koban L, Wager TD. The cognitive neuroscience of placebo effects: concepts, predictions, and physiology. Annu Rev Neurosci. 2017:40:167-88.

33. Tachibana $Y$, Narukawa M. Investigation of influencing factors on highe placebo response in East Asian versus Western clinical trials for partial epilepsy: a meta-analysis. Clin Drug Investig. 2013;33(5):315-24.

34. Allgaier M, Allgaier C. An update on drug treatment options of Alzheimer's disease. Front Biosci (Landmark Ed). 2014;19:1345-54.

35. Chen Z, Zhong C. Decoding Alzheimer's disease from perturbed cerebral glucose metabolism: implications for diagnostic and therapeutic strategies. Prog Neurobiol. 2013;108:21-43.

\section{Publisher's Note}

Springer Nature remains neutral with regard to jurisdictional claims in published maps and institutional affiliations. 